\title{
DESARROLLO DE LA NEUROCIENCIA EN LA FACULTAD DE CIENCIAS
}

\author{
Juan Bacigalupo Vicuña
}




\section{JUAN BACIGALUPO VICUÑA}

Se graduó como Licenciado en Biología de la Facultad de Ciencias de la Universidad de Chile en 1975 y como Doctor en Ciencias en Brandeis University, Estados Unidos, en 1983. Desde 1976 es miembro del Departamento de Biología de la Facultad de Ciencias, del cual es Profesor Titular. Desde 2013 es Presidente de la Sociedad Chilena de Neurociencia. Su trabajo se ha enfocado principalmente en la fisiología sensorial, con énfasis en los mecanismos de transducción en células fotorreceptoras, neuronas sensoriales olfatorias, de feromonas y termorreceptoras. También ha realizados estudios en el neuroepitelio y la amígdala olfatoria. 


\section{DESARROLLO DE LA NEUROCIENCIA EN LA FACULTAD DE CIENCIAS}

\section{INTRODUCCIÓN}

La neurociencia es una de las áreas más amplias de la ciencia y de mayor expansión e impacto en la actualidad. Esto resulta naturalmente del creciente interés por comprender el cerebro humano, impulsado por considerables avances alcanzados recientemente. Este objetivo representa un desafío monumental, puesto que sin duda alguna dicho órgano constituye la entidad más compleja que conocemos, además de ser lo que principalmente distingue a nuestra especie de todas las demás. El estudio del cerebro abarca necesariamente desde lo molecular hasta los aspectos más integrativos y complejos de la mente, tales como el conocimiento, la conciencia, la percepción, las emociones, el lenguaje y la memoria, entre otros. Es actualmente impensable aspirar a una comprensión integral del cerebro sin tomar en consideración y relacionar entre sí ambos niveles y todos aquellos comprendidos entre ellos, abarcando también complejísimos circuitos neuronales y sus interconexiones. Los enormes e impresionantes avances logrados por la biología molecular, estructural, celular, la biofísica, la fisiología, la neurobiología y otras disciplinas cuyos bordes parecían hasta hace pocos años muy definidos e independientes entre sí, tuvieron como consecuencia que estos gradualmente se fueron fundiendo hasta desaparecer. La brillante historia de la neurociencia en esta facultad es fiel reflejo de esta situación.

La Facultad de Ciencias tuvo un papel clave en el origen de la neurociencia en el país. Los primeros científicos que formaron su cuerpo académico, constituido en esta facultad en 1965, cultivaron la bioquímica (Hermann Niemeyer, Osvaldo Cori), la biología del desarrollo (Luis Izquierdo, Juan Fernández y Mario Luxoro) y la neurobiología (Humberto Maturana). Los primeros programas de doctorado que se ofrecieron tuvieron las menciones de bioquímica y de fisiología. Con el tiempo y respondiendo a la tendencia indicada arriba, estos se integraron en el programa de Biología Molecular, Celular y Neurociencia, como se sigue llamando. Mitzy Canessa tuvo un rol clave impulsando este paso a mediados de los años ochenta, siendo ella quien propuso el nuevo nombre del programa.

La biofísica partió en Chile con Mario Luxoro, quien a principios de los años sesenta reunió a un grupo de científicos jóvenes y talentosos y los formó bajo 
un perfil nuevo en Chile, donde se priorizaba una aproximación básica sólida y cuantitativa para abordar los problemas biológicos. Dicho grupo se enfocó en el estudio de la excitabilidad de las neuronas, para lo cual utilizaron el axón gigante de la jibia. Los famosos trabajos que habían sido recién publicados por Alan Hodgkin y Andrew Huxley, donde establecieron las bases electrofisiológicas del impulso nervioso y que fueron realizados en Inglaterra en el axón gigante de jibia (Loligo pealeii), inspiraron fuertemente al grupo de Luxoro. Esos estudios les valieron el Premio Nobel de Fisiología a esos dos investigadores británicos. Luxoro había regresado recientemente de los Estados Unidos tras doctorarse en el Massachusetts Institute of Technology (MIT), entusiasmado por la propuesta del gran biofísico norteamericano Kenneth Cole de instaurar en el país un laboratorio de electrofisiología donde se explotara el uso del axón de la jibia chilena (Dosidicus gigas), de mucho mayor tamaño que los de las especies del hemisferio norte. Esta particularidad ofrecía características experimentales extremadamente ventajosas en tiempos en que las técnicas disponibles estaban limitadas por las dimensiones de las células. Luxoro asumió el desafío, reclutó a su primer estudiante, Eduardo Rojas, y luego a Mitzy Canessa, Sigmund Fischer y Fernando Vargas, iniciando la extraordinaria aventura científica que llegó a constituir una Escuela de Biofísica de nivel mundial.

El grupo de Luxoro se instaló durante los veranos en el Instituto de Biología Marina de Montemar, perteneciente en ese entonces a la sede de Valparaíso de la Universidad de Chile. Allí montaron los equipos que ellos mismos construyeron, los que les fueron donados por sus colegas del extranjero y otros que adquirieron con un proyecto de la Fundación Rockefeller, e iniciaron sus investigaciones con un enorme entusiasmo. El intenso ritmo de trabajo de los biofísicos no se ajustaba al que se acostumbraba en el instituto que los albergaba, produciendo roces que entorpecieron severamente su trabajo. La situación se tornó insostenible, obligándolos a buscar un espacio propio donde pudieran funcionar normalmente. En 1962 Luxoro consiguió con el rector de la Universidad de Chile de la época, Eugenio González, financiamiento para adquirir una casa al frente del instituto, que pudiera ser adaptada para las necesidades de los biofísicos. El grupo creció rápidamente, se fueron integrando estudiantes y se incrementaron fuertemente las publicaciones, al tiempo que un buen número de importantes científicos del extranjero se vio atraído por este lejano laboratorio donde estaba generándose tan buen y abundante trabajo, y comenzaron a venir al país, sumándose al trabajo de los chilenos. Entre los prominentes biofísicos que pasaron por Montemar estuvieron Clay Armstrong, Richard Keynes, Robert Taylor, Gerry Ehrenstein e Ichiji Tasaki, entre varios otros. Estas personas aportaron muchísimo al grupo de Montemar, enriqueciendo la vida del laboratorio y ampliando el espectro de las investigaciones 
que allí se realizaban. Adicionalmente, los visitantes trajeron equipos que dejaron en el laboratorio para el beneficio de los investigadores locales. El aporte de esos investigadores a la formación de los jóvenes fue enorme.

El ritmo de trabajo en el laboratorio de Montemar era frenético. Un par de pescadores salían en la noche a atrapar las jibias y llegaban de vuelta con ellas muy temprano. Los experimentos empezaban de inmediato y se prolongaban hasta altas horas de la noche, para aprovechar al máximo los axones. La productividad fue muy alta, se publicaron los trabajos que de allí surgieron en las mejores revistas y su impacto fue inmenso, despertando el reconocimiento de la comunidad científica internacional. En 1967 el laboratorio fue invadido por el mar, provocando un desastre mayúsculo, pues el agua se llevó consigo una buena cantidad de equipos y dañó otros. Con la valiente ayuda del pescador José Soto lograron rescatar de las olas varios de ellos. Osciloscopios y otros equipos electrónicos fueron lavados con agua dulce y reparados por los mismos investigadores hasta que lograron montar nuevamente el laboratorio y reiniciar su trabajo con nuevos bríos.

La atmósfera que se vivía era muy especial y fue un factor determinante para el extraordinario nivel que se alcanzó en la formación de estudiantes. Un aspecto muy notable fue la libertad que se les daba a los estudiantes en la realización de sus investigaciones. No existían diferencias jerárquicas entre profesores y estudiantes, ni privilegios en el uso de los equipos. Esto alguna vez provocó roces con algunos visitantes extranjeros acostumbrados a un sistema más jerárquico, por lo cual no compartían esta manera tan particular de funcionar. Asimismo, los estudiantes podían publicar sus trabajos sin la coautoría de los investigadores establecidos, algo extremadamente inusual en la época y más aún hoy en día. Mario Luxoro impuso el sello de la generosidad y del reconocimiento de sus méritos personales, lo que acrecentó naturalmente su entusiasmo y los indujo a convertirse rápidamente en científicos independientes.

De ese laboratorio surgieron biofísicos muy prominentes, entre ellos Cecilia Hidalgo, Ramón Latorre, Francisco Bezanilla y Julio Vergara; todos ellos realizaron allí sus tesis doctorales. Los dos primeros, actualmente profesores de la Universidad de Chile y de Valparaíso, respectivamente, han sido galardonados con el Premio Nacional de Ciencias. Bezanilla, por su parte, es profesor de la Universidad de Chicago y es considerado entre los más destacados biofísicos del mundo, habiendo recibido numerosos reconocimientos internacionales del más alto nivel. Julio Vergara es también un reconocido investigador de la Universidad de California en Los Ángeles (UCLA). Siguieron otras generaciones que también han destacado en el campo de la biofísica y neurobiología y que a su vez han preservado el espíritu de Montemar en su trabajo experimental e impreso el sello de la escuela de Luxoro en la formación de estudiantes. 
En el laboratorio de Montemar se originaron aportes fundamentales a la fisiología celular. Así, por ejemplo, Luxoro y Rojas (1963) demostraron que los canales iónicos, que constituyen la base molecular de la excitabilidad, consisten en proteínas integrales de la membrana celular y no en los lípidos que constituyen dicha membrana, que era lo que hasta entonces se pensaba. Este trabajo, que representa un clásico de la literatura del campo de la biofísica, fue publicado por la revista Nature. Posteriormente, Luxoro y Rissetti (1967) reportaron por primera vez que el ión calcio es retenido en el interior de la célula, contrariamente a los demás iones de importancia electrofisiológica. Este concepto es hoy clave en la fisiología celular, pues ese ión tiene la particularidad de regular virtualmente todos los procesos fisiológicos que tienen lugar en las células. Hoy está muy bien establecido que las células poseen en su citoplasma sistemas de membranas internas que efectivamente almacenan el calcio de una manera dinámica, lo que les permite ejercer un finísimo control espacial y temporal de su concentración citoplasmática. Armstrong, Bezanilla y Rojas (1973) demostraron que el sodio y el potasio pasaban por la membrana a través de canales constituidos por proteínas diferentes, lo que en ese entonces estaba en discusión.

El golpe militar sacudió brutalmente al laboratorio de Montemar. Varios de sus integrantes estaban en la lista de las personas buscadas para ser detenidas y debieron esconderse y, en cuanto pudieron, exiliarse. Otros que no eran perseguidos también abandonaron el país buscando un entorno en que pudieran continuar haciendo ciencia, lo que aquí se tornó extremadamente difícil. Una muy grave y lamentable consecuencia de esta situación fue la suspensión del Doctorado en Fisiología, debido a que el éxodo de científicos redujo más allá de lo aceptable la masa crítica de académicos requeridos para poder sostener el programa. Esto provocó que los jóvenes interesados en proseguir su formación de postgrado debieran salir al extranjero. A mediados de los ochenta empezaron a retornar científicos chilenos, incluyendo varios jóvenes recién doctorados, lo que permitió revivir el programa de doctorado. Sin embargo, mientras tanto el laboratorio de Montemar pudo seguir funcionando, aunque muy dificultosamente, gracias a que Mario Luxoro optó por quedarse en Chile y luchar para impedir la muerte de la investigación en el país. Naturalmente el ritmo del laboratorio decayó dramáticamente, a pesar de lo cual Luxoro continuó formando estudiantes solitariamente y publicando en buenas revistas. Chile le debe mucho a Mario Luxoro, no solo por el papel que tuvo en la iniciación de la investigación en biofísica y su destacada labor como decano de la Facultad de Ciencias entre 1968 y 1972, sino que también en la sobrevivencia de ésta en tan difíciles condiciones.

Un hecho sorprendente que ocurrió en el año 1970 fue que la jibia despareció de los mares chilenos debido a un fenómeno natural que la impulsó a emigrar a 
mares desconocidos, de donde regresó muchos años después. Esto hizo necesario buscar otra fuente de investigación, que terminó siendo la célula muscular gigante del Megabalanus psittacus, comúnmente conocido en Chile como picoroco. Aquí se estudió la contracción muscular y el acoplamiento entre las señales eléctricas de la membrana celular y la contracción. Desde principios de los años noventa la actividad del laboratorio fue prácticamente nula. Sin embargo, hace pocos años Francisco Bezanilla retornó al laboratorio con Miguel Holgrem, chileno residente en Estados Unidos, y lo han estado haciendo periódicamente, dos o tres veces al año. Allí utilizan el axón de jibia, que sigue constituyendo una preparación incomparable para cierto tipo de estudios electrofisiológicos.

Algunos de los académicos que forman parte del Departamento de Biología de esta facultad derivan directa o indirectamente del laboratorio de Montemar. Osvaldo Álvarez, contemporáneo de Ramón Latorre y Cecilia Hidalgo y también un destacadísimo biofísico, se formaron también en esa misma escuela. Daniel Wolff fue también producto de Montemar. Cecilia Vergara y Juan Bacigalupo fueron parte de ese laboratorio en una tercera generación, mientras que Magdalena Sanhueza y Daniel Basilio representan a generaciones posteriores. Álvarez, Vergara, Bacigalupo, Sanhueza y Basilio son actualmente académicos que realizan su investigación en esta facultad. Todos ellos han continuado transmitiendo el sello de Montemar a las nuevas generaciones. Un extenso número de científicos provenientes de ese laboratorio se hallan repartidos por el mundo en prestigiosas universidades, así como en otras facultades de la Universidad de Chile y otras universidades del país.

Osvaldo Álvarez se incorporó muy joven como académico al Departamento de Biología, enfocándose en el estudio de los canales de iones, trabajo en que ha hecho importantes contribuciones, mundialmente reconocidas. Él también ayudó a mantener la actividad científica durante los oscuros años setenta acogiendo a varios estudiantes en su laboratorio, a quienes transmitió su espíritu crítico, rigurosidad y sólidos conocimientos de los fenómenos fundamentales relacionados con la excitabilidad celular. Se hizo cargo del curso de Biología Celular, que aún es recordado con admiración por aquellos que lo tomaron. Muchos de quienes actualmente trabajamos en esta facultad reconocemos en Osvaldo a uno de nuestros más importantes maestros, al igual que innumerables colegas que se encuentran en muchos otros lugares del planeta.

Otra rama de la neurociencia que tuvo un muy potente desarrollo en esta facultad fue establecida por Humberto Maturana. Contemporáneo de Luxoro, Maturana se interesó más bien por aspectos integrativos del sistema nervioso. Él inició su brillante carrera científica en Inglaterra y en la Universidad de Harvard, donde hizo su doctorado. Luego de pasar un tiempo en el MIT regresó a Chile cargado de nuevas ideas y habiendo realizado un trabajo tremendamente influyente y hasta hoy citado 
en la visión de los anfibios (Maturana y cols., 1960). Se instaló a tiempo completo en la Facultad de Ciencias apenas ésta fue habilitada con laboratorios para acoger a científicos de las más diversas disciplinas. En ese tiempo ya se había él aproximado al concepto de la autopoiesis a partir de ideas que había estado incubando y elaborando por muchos años (Maturana, 1969). En 1970 regresó a Chile Francisco Varela, muy joven y recientemente doctorado en la Universidad de Harvard y también muy lleno de ideas propias y revolucionarias para la época. Varela se instaló en el laboratorio de Maturana y juntos emprendieron una colaboración que ha sido de las más fructíferas en la ciencia chilena y de una sobresaliente estatura internacional. Aparte de realizar trabajo experimental en el sistema visual, en el cual ambos habían tenido independientemente sendas experiencias en sus respectivas permanencias en los Estados Unidos, se enfocaron en el problema que había inquietado a Maturana, la necesidad de definir lo que constituye un ser vivo. En ese entonces prevalecía la concepción del ser vivo como un ente que integraba una cantidad de propiedades, cuya lista no parecía tener un fin bien definido; además, se pensaba que el ser vivo constituía un sistema abierto que procesaba metabólicamente lo que recibía de su entorno. Maturana se había convencido de que esta definición era inadecuada. Para él un ser vivo podía ser mejor concebido como un sistema autónomo, capaz de generar su composición molecular y dinámica interna de manera independiente, es decir, un sistema cerrado. El término autopoiesis lo inventó Maturana derivándolo de la palabra griega poiesis, que significa producción, es decir, un ser vivo es una unidad capaz de generar autónomamente sus propios componentes. Esta idea fue tomando forma, derivando hacia una nueva manera de concebir los sistemas biológicos en general. Maturana y Varela trabajaron arduamente estas ideas durante los tres años que el segundo alcanzó a estar en el país, las elaboraron extensamente, mucho más allá del propósito original, abarcando otras dimensiones de los sistemas biológicos. Todo esto cristalizó en el libro "De máquinas y seres vivos. Autopoiesis: la organización de lo vivo", publicado por la Editorial Universitaria de la Universidad de Chile (Maturana y Varela, 1973). Este libro llegó a tener un enorme impacto a nivel mundial. Su influencia rebasó a la biología, siendo los conceptos allí vertidos incorporados no solo por otras ramas de la ciencia, sino que también por la filosofía y varias ramas de las ciencias sociales.

En su laboratorio se estudiaba el sistema visual a un gran nivel y de allí surgieron trabajos de mucha relevancia. El equipo formado por estos dos grandes científicos fue tremendamente influyente. Ellos sumaron a los estudios neurobiológicos una aproximación epistemológica y atrajeron a muchos jóvenes que se formaron con ellos. Maturana y Varela crearon un curso de Biología Celular para los estudiantes de primer año de Biología que fue tremendamente original, provocativo y también profundamente marcador. Quienes tuvimos la fortuna de haber tomado ese 
curso podemos dar fe de la trascendencia que este tuvo en nuestra formación científica. Tras la partida de Varela en 1973, Maturana creó el curso de Biología del Conocimiento, mediante el cual transmitió a sus alumnos sus ideas sobre los seres vivos, el sistema nervioso y el conocimiento de forma tan atrayente y convincente que estas imprimieron en ellos una forma de pensar los problemas biológicos que ha guiado sus investigaciones en las respectivas y muy variadas disciplinas que cada cual desarrolló posteriormente.

Francisco Varela abandonó el país tras el golpe militar pues su vida corría peligro, trasladándose primero a Costa Rica y después a los Estados Unidos. A principios de los años ochenta regresó a la Facultad de Ciencias por otros tres años. En su corta estadía atrajo a una cantidad de jóvenes estudiantes a su laboratorio, guiando sus memorias de Licenciatura en Biología. Entre ellos se encuentran Jorge Mpodozis, Juan Carlos Letelier y Gonzalo Marín, quienes actualmente comparten el Laboratorio de Neurobiología de esta Facultad, donde las concepciones de Maturana y Varela continúan ejerciendo su influencia y siguen siendo fuente de inspiración. Al igual que la escuela de Luxoro, la de Maturana sigue vigente y ha dado origen a decenas de científicos destacados con su sello característico, repartidos en numerosos lugares, tanto en Chile como en el exterior.

Durante su segundo período en Chile, Varela se unió nuevamente a Maturana para continuar desarrollando sus ideas epistemológicas más ampliamente, las cuales plasmaron en un nuevo libro, "El árbol del conocimiento", publicado igualmente por la Editorial Universitaria (Maturana y Varela, 1984). Ambos libros han sido traducidos a numerosos idiomas y permanecen vigentes hasta el día de hoy. Francisco Varela dejó definitivamente el país en 1985, instalándose en París, donde permaneció hasta su muerte en 2001. Sus contribuciones en los campos de la neurobiología, la inmunología, la epistemología, entre otros, tanto teóricas como experimentales, fueron y continúan siendo de una influencia enorme. Tuvo muchísimos discípulos y seguidores en todo el mundo. Varela adoptó el budismo, al cual se volcó con gran intensidad, incorporándolo a su pensamiento. En sus últimos años llegó a tener una cercana relación con el Dalai Lama, quien le encargó organizar algunas reuniones en que discutía ciencia en el contexto del budismo.

Por su parte, Maturana continuó en la Facultad de Ciencias hasta el día de hoy, siendo actualmente profesor emérito, al igual que Mario Luxoro. Ambos fueron galardonados con el Premio Nacional de Ciencias. Maturana dirige el Instituto de Formación Matríztica, en que ha aplicado destacadamente sus ideas hacia muy diversos ámbitos, como la psicología, la sociedad, el amor y otros.

Un acontecimiento que remeció muy fuertemente a la neurociencia en nuestro departamento fue un incendio ocurrido en el Laboratorio de Neurobiología en el año 2006, Este laboratorio, que estaba albergado en las antiguas barracas de madera, 
fue completamente destruido por el fuego. Como consecuencia de esta tragedia se perdieron instrumentos, preparaciones, resultados de tesis en marcha, aparte de material bibliográfico y una buena parte de objetos irrecuperables que representaban la rica historia de sus integrantes, desde Maturana en adelante. Afortunadamente se logró construir un laboratorio en su reemplazo, que prontamente reactivó sus actividades con mucha energía.

Aparte de lo anterior, en 1974 se radicó en la Facultad de Ciencias Orlando Gutiérrez, ingeniero eléctrico que luego de una permanencia en el MIT y en la Facultad de Medicina de esta Universidad formó un laboratorio para el estudio de la visión en peces. Gutiérrez formó a varios estudiantes, entre ellos a Julio Alcayaga, actualmente académico de esta facultad.

En el año 2002 se construyó el edificio actualmente conocido como Biología/ Milenio gracias al Instituto Milenio de Biología Celular y Biotecnología que existió entre los años 2000 a 2005 y que dirigió Ricardo Maccioni y del cual formaron parte varios académicos locales. Ese edificio albergó a varios de los laboratorios del Departamento de Biología en mucho mejores condiciones que en las barracas donde antes estaban ubicados. En años más recientes llegaron al Departamento de Biología numerosos investigadores interesados en distintos aspectos del sistema nervioso, constituyendo en el presente un $40 \%$ de sus académicos. Esto refleja la gran relevancia de la neurociencia en nuestro país. En este departamento se realizan estudios del sistema nervioso en biología celular (Christian González, Ricardo Maccioni, Tulio Núñez, Alejandro Roth, Elías Utreras), biología del desarrollo del sistema nervioso (Miguel Allende, Verónica Palma), fisiología celular (Osvaldo Alvarez, Julio Alcayaga, Juan Bacigalupo, Daniel Basilio, Ricardo Delgado, Magdalena Sanhueza, Cecilia Vergara) y neurobiología (Juan Carlos Letelier, Gonzalo Marín, Humberto Maturana, Jorge Mpodozis).

\section{REFERENCIAS}

Armstrong CM, Bezanilla F, Rojas E. Destruction of sodium conductance inactivation in squid axons perfused with pronase. The Journal of General Physiology 62: 375-391. 1973.

Luxoro M, Rissetti S. Calcium efflux in giant axons. Biochemica et Biophysica Acta 135: 368-370. 1967.

Luxoro M, Rojas E. Microinjection of trypsin into axons of squid. Nature 199: 78-79. 1963.

Maturana HR. Neurophysiology of congnition. En Cognition a Multiple View. Editor Paul Garvin, Spartan Books, 1969. 
Maturana HR, Lettvin J, McCulloch W, Pitts W. Anatomy and physiology of vision in the frog.

Journal of General Physiology 43: 129-175, 1960.

Maturana HR, Varela FJ. De máquinas y seres vivos. Autopoiesis: la organización de lo vivo. Editorial Universitaria. Chile. 1973. 74 pp.

Maturana HR, Varela FJ. El árbol del conocimiento. Editorial Universitaria. Chile. 1984. $171 \mathrm{pp}$. 